\title{
Effect of Acupuncture on Polycystic Ovary Syndrome in Animal Models: Study Protocol for a Systematic Review and Meta-Analysis
}

\section{Jinjin Gao}

First Affiliated Hospital, Heilongjiang University of Chinese Medicine

\section{Wei Li}

Heilongjiang University of Traditional Chinese Medicine: Heilongjiang University of Chinese Medicine

Yangyang Li

Heilongjiang University of Chinese Medicine

Yan Li ( $\Delta$ liyantcm@163.com )

First Affiliated Hospital, Heilongjiang University of Chinese Medicine, 26 Heping Road, Harbin, 150040, China https://orcid.org/0000-0001-8915-9330

\section{Protocol}

Keywords: Polycystic ovary syndrome, Acupuncture, Animal model, protocol, Systematic review

Posted Date: January 15th, 2021

DOl: https://doi.org/10.21203/rs.3.rs-144670/v1

License: (c) (i) This work is licensed under a Creative Commons Attribution 4.0 International License. Read Full License 


\section{Abstract}

Background: Polycystic ovary syndrome (PCOS) is one of the most common endocrine disorders among women of reproductive age. As a widely used complementary and alternative therapy, acupuncture is increasingly used to treat PCOS. However, the effect of acupuncture in treating PCOS is uncertain and the mechanisms are unclear. This systematic review aims to determine the efficacy of acupuncture on PCOS in animal preclinical models.

Methods: We will search the following databases: PubMed, Web of Science, China National Knowledge Infrastructure and Chinese Science and Technology Periodical Database. We will only include animal experiments of acupuncture in treating PCOS. The primary outcome will be homeostatic model assessment- insulin resistance. The risk of bias will be assessed using the Systematic Review Centre for Laboratory Animal Experimentation (SYRCLE) risk of bias tool. Confidence in the cumulative evidence will be assessed using the Grading of Recommendations Assessment, Development and Evaluation (GRADE) approach. All meta-analyses will be conducted using Review Manager 5.4.

Discussion: To the best of our knowledge, the use of acupuncture in treating PCOS has not yet been systematically reviewed in animal models. The evidence generated from this systematic review and metaanalysis could benefit future researches.

Systematic review registration: OSF (Registration DOI: 10.17605/OSF.IO/FNM37)

\section{Background}

Polycystic ovary syndrome (PCOS) is one of the most common reproductive endocrine disorders in women of reproductive age, affecting $5-20 \%$ of the reproductive age women globally [1].

Since the balance of the hypothalamic-pituitary-ovarian (HPO) axis is disrupted, PCOS is characterized by chronic ovulation disorder, hyperandrogenemia, and bilateral polycystic ovaries. Increased luteinizing hormone (LH) and anti-mullerian hormone (AMH) are commonly observed in PCOS women [2]. PCOS impairs both endocrine and metabolic functions, insulin resistance is always present in PCOS women regardless of body weight [2].

Acupuncture originates from ancient China and has been applied for thousands of years. It is now widely recognized as a complementary and alternative therapy in current clinical practice. There are existing systematic reviews on acupuncture for PCOS [3-5], however, the quality of methodological and outcomes are discrepant. A recent updated Cochrane review [3] and Jo et.al [4] both reported very limited evidence for acupuncture in improving ovulation, menstruation rates and hormonal changes versus sham acupuncture. Wu et.al performed meta-analysis including 22 RCTs with 2315 subjects, showed that acupuncture could promote the recovery of menstrual cycles and regulate the levels of hormone in patients with PCOS [5]. 
The effect of acupuncture on PCOS-like animal models were investigated. Acupuncture bidirectionally regulates the HPO axis and modulates sex hormone levels [6, 7]. It might modulate the reproductive and endocrine function of PCOS via sympathetic nerve stimulation [8]. It is proved that acupuncture could play an important role in the regulation of hormone levels, ovulation induction, psychological regulation, and weight control $[9,10]$. Also, experiments on PCOS animal models showed that electrical acupuncture (EA) ameliorated insulin resistance and mitochondrial dysfunction by promoting autophagy [11]. EA regulates ovarian innervations to increase the numbers of preovulatory follicles in PCOS-like rats [12]. Both low-frequency EA and manual acupuncture restored disturbed oestrous cyclicity while EA decreases serum testosterone level in PCOS-like rats [13].

Animal experiment serves as a bridge between basic research and clinical practice, which is an essential component of pre-clinical research and evidence-informed. The systematic reviews and meta-analysis of animal experimental can retrospectively compare the appropriateness of the animal models and reduce the risk for the translation of animal experiments to the clinical practice [14-16]. Therefore, systematic reviews and meta-analysis of animal studies play an important role.

The objective of the proposed systematic review and meta-analysis is to assess the effectiveness of acupuncture in treating PCOS animal model and provides evidence to inform clinical practice and decision-making.

\section{Methods}

\section{Protocol and registration}

This protocol follows the preferred reporting items for systematic reviews and meta-analysis protocols (PRISMA-P) guidelines (Additional file 1) [17] and is adapted from the structure provided in the Systematic Review Protocol for Animal Intervention Studies [18]. This protocol was registered on OSF (Registration DOI: 10.17605/OSF.IO/FNM37).

\section{Eligibility criteria}

\section{Types of studies}

This systematic review will include both randomized and non-randomized controlled studies evaluating the effect of acupuncture on animal models of PCOS. There will be no restriction on language, date, or publication status.

\section{Types of animal models}

This systematic review will examine all animal models of PCOS regardless of species or size of the animal.

\section{Types of comparators}


The comparison group will include PCOS animals induced by the same method as the intervention group but have not undergone intervention.

\section{Types of intervention}

We will include studies using both traditional acupuncture and contemporary acupuncture (in which needles were not inserted in classical meridian points). We will adopt hand stimulation, electrical stimulation, or warming needle with moxibustion. Acupuncture without needling will be excluded such as acupressure, acupoint injection, tap-pricking, and cupping.

\section{Exclusion criteria}

We will exclude case reports, cohort studies, congress abstracts, letters to editors, in vitro experiments, and human studies. Studies comparing animal models to healthy controls will also be excluded.

\section{Types of outcome measures}

Preliminary searches have shown that parameters concerning hormone and insulin resistance have been reported in experimental studies of acupuncture in treating PCOS animal models. The following outcome measures will be used:

I. Primary outcome: homeostatic model assessment- insulin resistance (HOMA-IR: (fasting insulin $(\mu \mathrm{U} / \mathrm{mL}) \times$ fasting glucose $(\mathrm{mmol} / \mathrm{L})) / 22.5)$.

II. Secondary outcome: testosterone (T), LH, LH/follicle stimulating hormone (FSH) ratio, AMH, body mass index (BMI: calculated using weight $(\mathrm{kg}) /$ height $\left(\mathrm{m}^{2}\right)$ ).

\section{Search and study identification}

The following sources will be searched: PubMed, Web of Science, China National Knowledge Infrastructure (CNKI), Chinese Science and Technology Periodical Database (VIP). The main terms "Polycystic ovary syndrome", "Acupuncture", and "Animal Experimentation", indexed in the MeSH system, will be combined. We will search the terms and their synonyms separately first and then combine and cross the search terms. Details of the Pubmed search strategy present in Additional file 2.

Two independent reviewers (JJG and YYL) will screen titles and abstracts for eligibility. Disagreements between reviewers will be resolved by third review (YL). Full texts will be obtained and evaluated by the same reviewers using a pre-designed form with inclusion and exclusion criteria. For duplicated publication, we will use the paper with more complete data. The study selection progress will be presented using PRISMA flow diagram (Additional file 2).

\section{Data collection}

Two reviewers (YL and JJG) will extract data independently using a pre-approved form. The following information will be recorded: study design, characteristics of included, characteristics of animal model, 
details of the intervention, and outcome measures. If the data is not available in the text but mentioned in the manuscript, the reviewer will contact the authors via email.

\section{Risk of bias assessment}

Risk of bias will be assessed by the SYRCLE's tool for assessing risk of bias (SYRCLE ROB)[19], which includes domains for selection bias (sequence generation, baseline characteristics, allocation concealment), performance bias (random housing and blinding), detection bias (random outcome assessment and blinding), attrition bias, reporting bias, and other biases.

\section{Data synthesis}

Data will be presented in summary tables to describe the study population and intervention. We will perform a meta-analysis using random effects models. Mean differences (MD) among groups with their respective $95 \%$ confidence intervals and $p$ values will be presented in forest plots. Data will be analyzed using Review Manager (RevMan) 5.4. The presence of heterogeneity will be evaluated by $\mathrm{I}^{2}$ and chisquare statistical analyses. Sources of heterogeneity will be explored by sensitivity analysis via systematic removal of individual trials. We will construct summary tables and provide a narrative synthesis if quantitative synthesis is not appropriate. Funnel graphics will be performed to evaluate publication bias if there are enough studies included.

We will use the Grading of Recommendations Assessment, Development and Evaluation (GRADE) approach [20] to assess the quality of evidence generated from each outcome. The GRADE contains five domains including risk of bias, inconsistency (heterogeneity), indirectness, imprecision, and publication bias. Two reviewers will assess the quality independently and the quality will be graded as "high", "moderate", "low", or "very low.

\section{Discussion}

To our knowledge, many experiments were performed to assess the effect of acupuncture in treating PCOS animal models, however, none have performed a systematic review and meta-analysis to evaluate overall findings. This meta-analysis will synthesize all the available findings on the effect of acupuncture therapy forPCOS animal experiments. The evidence would contribute to develop more precise preclinical experiments and offer future perspectives in this field.

\section{Abbreviations}

PCOS: Polycystic ovary syndrome; SYRCLE: Systematic Review Centre for Laboratory Animal Experimentation; GRADE: Grading of Recommendations Assessment, Development and Evaluation; HPO: hypothalamic-pituitary-ovarian; LH: luteinizing hormone; $\mathrm{AMH}$ : anti-mullerian hormone; EA: electrical acupuncture; PRISMA-P: preferred reporting items for systematic reviews and meta-analysis protocols; CNKI: China National Knowledge Infrastructure; VIP: Chinese Science and Technology Periodical 
Database; HOMA-IR: homeostatic model assessment; T: testosterone; FSH: follicle-stimulating hormone, BMI: body mass index; s; MD: Mean differences; RevMan: Review Manager.

\section{Declarations}

\section{Ethics approval and consent to participate}

Not applicable

\section{Consent for publication}

Not applicable

\section{Availability of data and materials}

Not applicable

\section{Competing interests}

The authors declare that they have no competing interests.

\section{Funding}

This study was supported in part by the National Foundation of Natural Science of China (grant number: 81804139), and Heilongjiang Provincial Administration of Traditional Chinese Medicine (grant number: ZHY2020-130) funded to Dr. Yan Li.

\section{Authors' contributions}

$Y L$ is the guarantor of the review. YL, JJG and WL conceived and designed the review. YL and JJG drafted the protocol. WL reviewed the draft added necessary comments. YYL designed search strategies. All authors read, reviewed, and approved the final manuscript.

\section{Acknowledgments}

None

\section{References}

1. Holton S, Hammarberg K, Johnson L. Fertility concerns and related information needs and preferences of women with PCOS. Hum Reprod Open. 2018;4:hoy019.

2. Patel S. Polycystic ovary syndrome (PCOS), an inflammatory, systemic, lifestyle endocrinopathy. J Steroid Biochem Mol Biol. 2018;182:27-36. 
3. Lim CED, Ng RWC, Cheng NCL, Zhang GS, Chen H. Acupuncture for polycystic ovarian syndrome. Cochrane Database Syst Rev. 2019;7:CD007689.

4. Jo J, Lee YJ, Lee H. Acupuncture for polycystic ovarian syndrome A systematic review and metaanalysis. Medicine. 2017;96(23):e7066.

5. Wu J, Chen D, Liu N. Effectiveness of acupuncture in polycystic ovary syndrome: A systematic review and meta-analysis of randomized controlled trials. Medicine (Baltimore). 2020;99(22):e20441.

6. Johansson J, Stener-Victorin E. Polycystic ovary syndrome: effect and mechanisms of acupuncture for ovulation induction. Evid Based Complement Alternat Med. 2013:762615.

7. Leonhardt H, Hellström M, Gull B, Lind A, Nilsson L, Janson PO, et al. Serum anti-Müllerian hormone and ovarian morphology assessed by magnetic resonance imaging in response to acupuncture and exercise in women with polycystic ovary syndrome: secondary analyses of a randomized controlled trial. Acta Obstet Gynecol Scandm. 2015;94(3):279-87.

8. Mannerås L, Cajander S, Lönn M, Stener-Victorin E.Acupuncture and exercise restore adipose tissue expression of sympathetic markers and improve ovarian morphology in rats with dihydrotestosterone-induced PCOS. Am J Physiol Regul Integr Comp Physiol. 2009;296(4):R1124-31.

9. Maliqueo M, Benrick A, Alvi A, Johansson J, Sun M, Labrie F, et al. Circulating gonadotropins and ovarian adiponectin system are modulated by acupuncture independently of sex steroid or $\beta$ adrenergic action in a female hyperandrogenic rat model of polycystic ovary syndrome. Mol Cell Endocrinol. 2015;412:159-169.

10. Maliqueo M, Benrick A, Marcondes RR, Johansson J, Sun M, Stener-Victorin E. Acupuncture does not restore metabolic disturbances in the P450 aromatase inhibitor induced-polycystic ovary syndrome rat model. Experimental physiology. 2017;102:113-127.

11. Peng Y, Guo L, Gu A, Shi B, Ren Y, Cong J, et al. Electroacupuncture alleviates polycystic ovary syndrome-like symptoms through improving insulin resistance, mitochondrial dysfunction, and endoplasmic reticulum stress via enhancing autophagy in rats. Molecular Medicine, 2020;26(1):73.

12. Tong X, Liu Y, Xu X, Shi J, Hu W, Ma T,et al. Ovarian Innervation Coupling With Vascularity: The Role of Electro-Acupuncture in Follicular Maturation in a Rat Model of Polycystic Ovary Syndrome. Frontiers in Physiology, 2020;11:474.

13. Feng $Y$, Johansson J, Shao R, Mannerås-Holm L, Billig H, Stener-Victorin E. Electrical and manual acupuncture stimulation affect oestrous cyclicity and neuroendocrine function in an $5 \mathrm{a}-$ dihydrotestosterone-induced rat polycystic ovary syndrome model. Exp Physiol. 2012;97(5):651-62.

14. Hooijmans $C$ R $\$ Ritskes-Hoitinga M. Progress in using systematic reviews of animal studies to improve translationalresearch. Plos Med. 2013;10(7): e1001482.

15. Ioannidis JP: Evolution and Translation of Research Findings:From Bench to Where?. Plos Clin Trials. 2006;1(7):e36.

16. Ritskes-Hoitinga M, Leenaars M, Avey M, Rovers M, Scholten R. Systematic reviews of preclinical animal studies can make significant contributions to health care and more transparent translational medicine. Cochrane Database of Systematic Reviews. Version 3. 2014. 
17. Shamseer L, Moher D, Clarke M, Ghersi D, Liberati A, Petticrew M, et al. Preferred reporting items for systematic review and meta-analysis protocols (PRISMA-P) 2015: elaboration and explanation. BMJ. 2015;35:g7647.

18. De Vries RBM, Hooijmans CR, Langendam MW, Van Luijk J, Leenaars M, Ritskes-Hoitinga M, et al. A protocol format for the preparation, registrationand publication of systematic reviews of animal intervention studies. Evid-Based Preclinical Med. 2015;2:1-9.

19. Hooijmans CR, Rovers MM, de Vries RB, Leenaars $M$, Ritskes-Hoitinga M, Langendam MW. SYRCLE's risk of bias tool for animal studies. BMC Med Res Methodol. 2014;14:43.

20. Guyatt G, Oxman AD, AkI EA, Kunz R, Vist G, Brozek J, et al. GRADE guidelines: 1. Introduction GRADE evidence profiles and summary of findings tables. J Clin Epidemiol. 2011;64:383-94.

\section{Supplementary Files}

This is a list of supplementary files associated with this preprint. Click to download.

- additionalfile1PRISMAP.pdf

- additionalfile2.pdf 